\title{
Analysis of $i c e A$ genotypes in South African Helicobacter pylori strains and relationship to clinically significant disease
}

\author{
M Kidd, R M Peek, A J Lastovica, D A Israel, A F Kummer, J A Louw
}

\begin{abstract}
Background-South African Helicobacter pylori isolates are characterised by the universal presence of $\operatorname{cag} A$ but have differences in vacuolating cytotoxin gene (vacA) alleles which correlate with clinically significant disease. However, the candidate virulence marker gene $i c e A$ has not been investigated.

Aim-To characterise the genetic organisation and heterogeneity of $i c e A$ genotypes in different South African clinical isolates. Patients and methods-We studied $H$ pylori strains isolated from 86 dyspeptic patients (30 with peptic ulcer disease (PUD), 19 with distal gastric adenocarcinoma (GC), and 37 with non-erosive gastritis) for the presence of iceA1 or $i c e A 2$ genes, and for differences in the genetic organisation of $i c e A 2$ by polymerase chain reaction, Southern hybridisation analysis, and sequencing.

Results-Genetic analysis of iceA1 demonstrated significant homology (92-95\%) with the USA type strain 26695 and probably functions as a transcriptional regulator, while a novel variant (iceA2D') of $i c e A 2$ and marked differences in predicted protein secondary structure of the ice $A 2$ protein were defined. iceA1 was detected in $68 \%$ and ice $A 2$ in $80 \%$ of all clinical isolates. Although approximately $40 \%$ of patients had both strains, a higher prevalence $(p<0.01)$ of GC patients were infected with iceA1 isolates which were invariably vacA s1/iceA1 $(\mathrm{p}<0.005 v$ gastritis). Isolates from PUD patients were distinguished by the structurally altered ice $A 2 D$ variant $(53 \% ; p<0.03 v$ gastritis) while the iceA2C variant distinguished isolates from patients with gastritis alone (67\%; p $<0.005 v$ PUD).
\end{abstract}

Conclusion-In this study, an association between iceA1 and GC was noted while differences in variants of $i c e A 2$ differentiated between PUD and gastritis alone. Combination analyses of ice $A$ genotypes and $v a c A$ alleles supported these associations.

(Gut 2001;49:629-635)

Keywords: adenocarcinoma; gastritis; Helicobacter pylori; ice A; peptic ulceration; protein prediction; sequencing; South Africa

Helicobacter pylori induces gastric inflammation in virtually all colonised individuals and such gastritis increases the risk of peptic ulcer disease (PUD) and non-cardia/distal gastric adenocarcinoma (GC). ${ }^{1-3}$ However, only a minority of patients carrying $H$ pylori develop clinical sequelae, suggesting that particular bacterial products may contribute to pathogenesis. ${ }^{4}$ Recently, a novel $H$ pylori gene $i c e A$ was identified following transcriptional upregulation on contact with gastric epithelial cells. ${ }^{5}$ ice $A$ exists as two distinct genotypes, ice $A 1$ and $i c e A 2$, and only ice $A 1$ RNA is induced following adherence in vitro. ${ }^{5} \mathrm{H}$ pylori ice $A 1$ demonstrates strong homology to a restriction endonuclease nlaIIIR in Neisseria lactamica, ${ }^{6}$ and in vivo carriage of $H$ pylori iceA1 strains has been reported to be associated with peptic ulceration and enhanced acute neutrophilic infiltration. ${ }^{58}$ However, linkage between the iceA1 genotype and ulcer disease is not universal, ${ }^{9}$ and thus may be population dependent.

In contrast with $i c e A 1$, ice $A 2$ has no significant homology to known proteins and its structure reveals patterns of repeated protein cassettes. Recently, the genetic organisation and sequence heterogeneity of ice $A 2$ has been studied, ${ }^{6}$ revealing five distinct ice $A 2$ subtypes. While ice $A 2$ strains are more prevalent among patients with asymptomatic gastritis and nonulcer dyspepsia, ${ }^{7}$ a statistically significant relationship between iceA2 subtypes and disease has not yet been defined.

South African $H$ pylori isolates are characterised by the universal presence of $\operatorname{cag} A$ but differences in the $3^{\prime}$ region of $c a g A$ and $v a c A$ alleles correlate significantly with clinical disease. ${ }^{10}{ }^{11}$ Our hypothesis was that $H$ pylori isolates from South African patients may also exhibit variability in their ice $A$ alleles and that this variability may be related both to clinical outcome and to vacA allelic status.

\section{Materials and methods}

$H$ PYLORI STRAINS AND DNA

$H$ pylori isolates, cultured from single biopsies from 86 clinically defined patients (37 patients with gastritis alone, 30 patients with PUD, and 19 patients with GC), were examined. Fifty six of the resultant isolates had vacA and $\operatorname{cag} A$ determined in a previous study. ${ }^{11}$ Putative clonal isolates were obtained from eight of the parent strains by serially reculturing single colonies twice. Reference strains 26695 (iceA1)

Abbreviations used in this paper: PUD, peptic ulcer disease; GC, gastric adenocarcinoma; PCR, polymerase chain reaction; SDS, sodium dodecyl sulphate; DAS, dense alignment surface; SSC, sodium chloride/sodium citrate. 


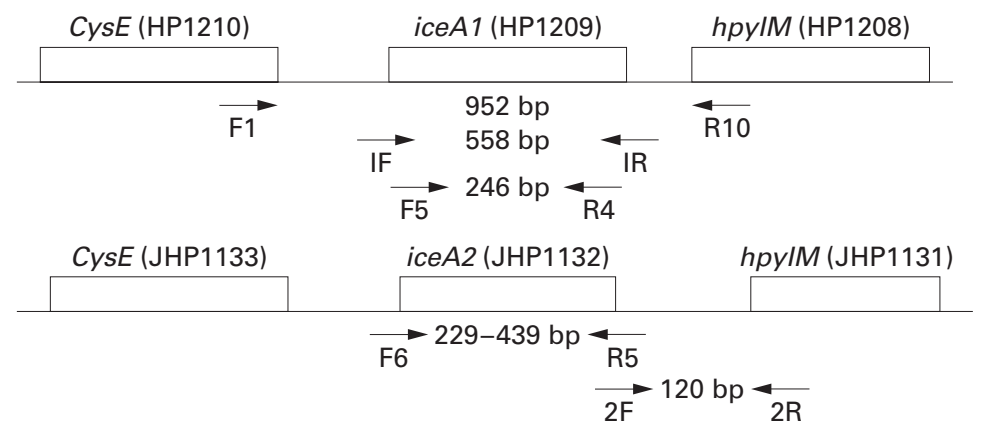

Figure 1 Primers, sites, and amplicon sizes for iceA1 (HP1209, GenBank Accession No AE000511) and iceA2 (FHP1132, GenBank Accession No AE001541) (see text for details).

isolated from a patient in the UK with gastritis $^{12}$ and $\mathrm{J} 99$ (iceA2) isolated from a USA patient with duodenal ulcer disease ${ }^{13}$ were used as ice $A 1$ and $i c e A 2$ positive and negative controls, respectively, in this study.

AMPLIFICATION OF iceA BY PCR

Polymerase chain reactions (PCRs) were performed as described previously ${ }^{511}$ but a final $\left[\mathrm{MgCl}_{2}\right]$ concentration of $3.5 \mathrm{mM}$ was used for the iceA1 PCRs. For amplification of the iceA1 allele, forward primer IceA1F (5'-CGTTGG GTAAGCGTTACAGAATTT) and reverse primer IceA1R (5'-TCATTGTATATCCTAT CATTACAAG) yielded a fragment of $558 \mathrm{bp}$ (fig 1). For ice $A 2$, primers $I c e A 2 F$ (5'-GTTG TCGTTGTTTTAATGAA) and IceA2R (5'GTCTTAAACCCCACGATTAAA) yielded a fragment of $120 \mathrm{bp}$. PCR was performed in a Sprint PCR (Hybaid, South Africa) under the following conditions: four minutes of preincubation at $94^{\circ} \mathrm{C}$ followed by 30 cycles of one minute at $94^{\circ} \mathrm{C}$, one minute at $62^{\circ} \mathrm{C}$ (iceA1) or $53^{\circ} \mathrm{C}$ (ice $A 2$ ), and one minute at $72^{\circ} \mathrm{C}$. Final extension was performed for 10 minutes at $72^{\circ} \mathrm{C}$.

Additional genotype specific PCR assays that used primers for iceA1 (IceA1F5 (5'-GTG TTTTTAACCAAAGTATC) and IceA1R4 (5'-CTATAGCCASTYTCTTTGCA)) and primers flanking the ice $A 2$ internal cassette were performed to confirm the presence of the genes and to identify the size and subtype of the iceA2 allele. ${ }^{7}$ Primers IceA2F6 (5'-GTTG GGTATATCACAATTTAT) and IceA2R5 (5'TTRCCCTATTTTCTAGTAGGT) yielded a fragment of 229,334 , or $439 \mathrm{bp}$ according to the existence of repeated sequences of 105 nucleotides. PCR was performed under the following conditions: four minutes of preincubation at $94^{\circ} \mathrm{C}$ followed by 30 cycles of one minute at $94^{\circ} \mathrm{C}$, one minute at $53^{\circ} \mathrm{C}$ (ice $A 1$ and iceA2), and one minute at $72^{\circ} \mathrm{C}$. Final extension was performed for 10 minutes at $72^{\circ} \mathrm{C}$. ice $A$ amplimers were examined by electrophoresis on $1 \%$ agarose gels according to standard procedures. ${ }^{11}$

SEQUENCING OF iceA1 AND iceA2

The complete iceA1 gene, from the upstream cysE (HP1210) to the downstream hypIM (HP1208), was amplified using F1 (5'-GGG TGCGATTTGCGTGGGCGATG) and R10 (5'-GATCATGGCCTACAACCGCATGGA), as described previously, ${ }^{14}$ while ice $A 2$ was amplified using primer sets IceA2F6/R5. PCR products were gel extracted (QIAEX II gel extraction kit; Qiagen, Cape Town, South Africa) and sequenced on an ABI PRISM 377 automated sequencer (ABI, Foster City, California, USA) using the ABI PRISM BigDye terminator cycle sequencing reagent kit with AmpliTaq DNA polymerase FS (PE Biosystems, Johannesburg, South Africa), as described previously. ${ }^{15}$ PCR and direct sequencing were performed at least twice to determine DNA sequences for each strain.

SOUTHERN HYBRIDISATION ANALYSIS

Southern hybridisation analysis was performed essentially as described previously. ${ }^{16}$ Chromosomal DNA digested with $B g l I I$ was subjected to electrophoresis on a $0.8 \%$ Tris acetate/EDTA agarose gel, transferred to a Hybond- $\mathrm{N}^{+}$membrane (AP Biotech, Piscataway, New Jersey, USA) by capillary transfer, and UV cross linked. Prehybridisation was performed using $6 \times$ sodium chloride/sodium citrate (SSC), $5 \times$ Denhardts, $0.5 \%$ sodium dodecyl sulphate (SDS), and $100 \mu \mathrm{g} / \mathrm{ml}$ of sheared salmon sperm DNA at $60^{\circ} \mathrm{C}$ for three hours. Buffer was replaced with fresh buffer containing denatured probe and hybridisation proceeded at $60^{\circ} \mathrm{C}$ for 18 hours. Membranes were washed for five minutes with $2 \times$ SSC, $0.5 \%$ SDS at room temperature, 15 minutes with $2 \times$ SSC, $0.1 \%$ SDS at room temperature, two hours with $0.2 \%$ SSC, $0.5 \%$ SDS at $60^{\circ} \mathrm{C}$, and 30 minutes with $0.2 \%$ SSC, $0.5 \%$ SDS at $60^{\circ} \mathrm{C}$. The membranes were then subjected to autoradiography for detection.

Probes for $i c e A 1$ and $i c e A 2$ were generated by PCR using primer pairs IceA1F5/IceA1R4 and IceA2F6/IceA2R5, respectively, as described previously, ${ }^{7}$ with 26695 and J99 chromosomal DNA as respective templates. Products were purified using QiaQuick PCR purification columns (Qiagen, Valencia, California, USA) and labelled with $\left[\alpha-{ }^{32} \mathrm{P}\right] \mathrm{dCTP}$ using the PRIME-IT RmT labelling kit as recommended by the manufacturer (Stratagene, La Jolla, California, USA). Unincorporated nucleotides were removed using ProbeQuant G-50 microcolumns according to the manufacturer's instructions (AP Biotech). Probe was denatured by boiling for five minutes and then placed on ice prior to hybridisation.

\section{COMPUTER ANALYSIS}

DNA sequences were analysed using the National Center for Biotechnology Information (NCBI) server (USA) and consensus secondary structure prediction for proteins was obtained using the META Predict Protein (which includes the SignalP, JPRED, TopPred, and DAS Servers (Columbia University, USA)).$^{17-20}$ SignalP predicts the presence and location of signal peptide cleavage sites, JPRED predicts secondary structures, TopPred is useful for prediction of the location and orientation of transmembrane sequences through the use of hydrophobicity patterns and by applying the "positive inside" rule, while DAS predicts the location of transmembrane using hydrophobicity patterns. Internet based 

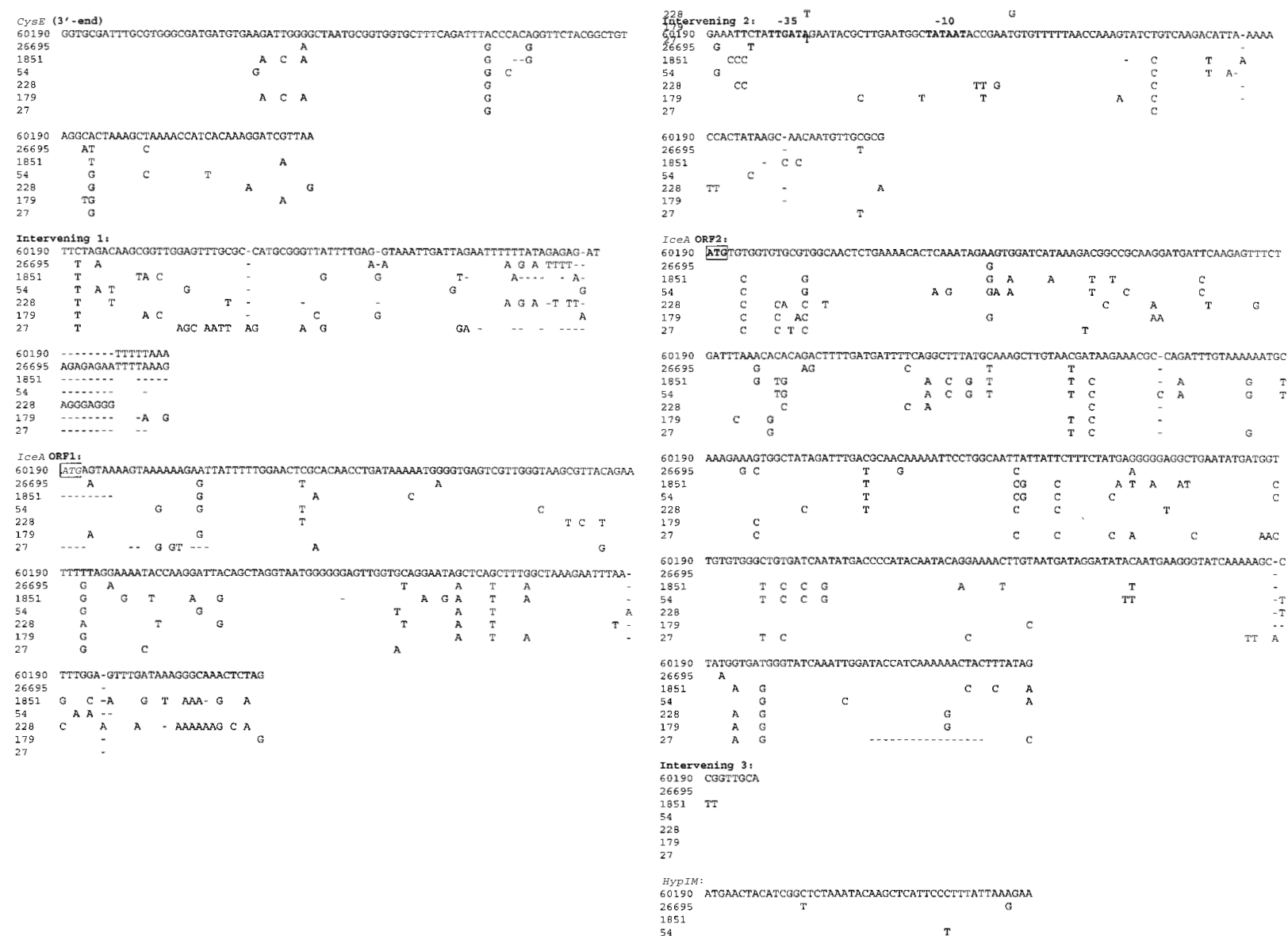

Figure 2 Alignment of nucleotide sequences of 60190, 26695, and five South African strains showing the 3' end of cysE, the complete iceA1 gene (ORF1 and ORF2), intervening sequences (1-3), and the $5^{\prime}$ end of hypIM putative start codon. ATG . (italicised and boxed) is the start codon for nlaIIIR and is only conserved in 3/5 strains. None of the strains however is predicted to produce the NLAIII homologue of 228 amino acids. ATG ${ }_{019}$ (boxed/bold), the start codon for iceA1, is conserved in all five South African isolates, as are the upstream transcription boxes (at-35 and-10). Predicted iceA1 proteins range in size from 128 to 136 amino acids, except for strain 54 (frameshift results in a protein of 53 amino acids). All isolates shared substantial protein homology (93-96\%) with HP1209. Sequence gaps are indicated by dashes (-).

searches were preformed at NCBI, the Institute for Genome Research (TIGR, Maryland, USA), and Astra-Zeneca (Boston, USA).

STATISTICS

Data were examined using the $\chi^{2}$ test or Fisher's exact test as appropriate. Probability levels of $<0.05$ were considered statistically significant.

\section{Results}

iceA1 SEQUENCE ANALYSIS

In a preliminary study, in order to establish the composition of ice $A$ gene products in South African $H$ pylori isolates, iceA1 (primers F1/R10: $952 \mathrm{bp}$ ) and iceA2 (primers F6/R5: 229-334 bp) were sequenced.

Five isolates with the iceA1 genotype (two from patients with gastritis alone, one from a patient with peptic ulceration, and two from patients with gastric cancer) were sequenced and compared with the type strains 60190 (GenBank Accession No U43917) and 26695. Alignment of these sequences revealed the presence of a putative conserved initiation codon at position $\mathrm{ATG}_{919}$ in all five South African strains as well as conserved upstream transcription initiation sites, as previously reported ${ }^{6}$ (fig 2). Predicted proteins ranged from the expected 128 amino acids to 136 amino acids in four strains. No full length NLAIII homologues were predicted. These results suggested that the iceA1 gene does not encode a functional restriction endonuclease but probably acts as a transcriptional regulator. ${ }^{14}$

iceA2 SEQUENCE ANALYSIS

Seven isolates with the $i c e A 2$ genotype (two from patients with gastritis alone, two from patients with PUD, and three from patients with GC) were sequenced and compared with the predicted gene (JHP1132) present at the same locus in strain J99 (fig 3).

Prior to our analysis, five different variants (A-E) based on size $(24,59,94$, or 129 amino acids) and/or sequence of ice $A 2$ had been described. ${ }^{6}$ The iceA2 gene (JHP1132) from strain J99 which conforms to the structure of repeats (14-13-16 $\left.6_{2 \mathrm{~B}}-6-10\right)$ demonstrated by Figueiredo and colleagues ${ }^{6}$ generates a predicted protein of 59 amino acids and is an example of the $i c e A 2 B$ subtype. ${ }^{6}$ None of the South African isolates sequenced in this series had either a 2 A structure (14-13-16-6-10) or a 


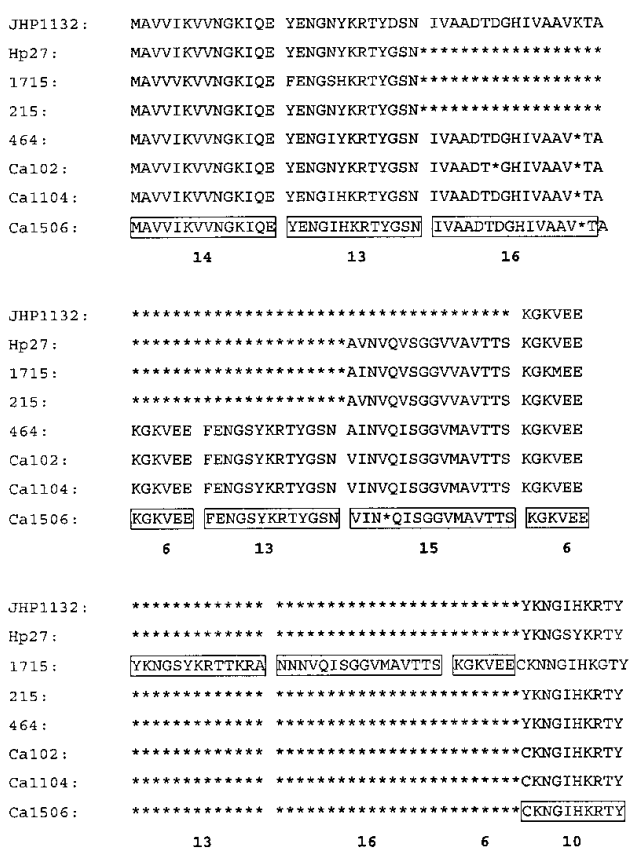

Figure 3 Amino acid alignment of ice $A 2$ genotypes from seven South African isolates and homology with $\mathcal{F H P 1 1 3 2 . ~}$ The different peptide domains are boxed and the amino acid number indicated. Asterisks $\left.{ }^{\star}{ }^{\star}\right)$ represent gaps in sequence homology.

2B structure. Two isolates ( $\mathrm{Hp} 27$ and 215) however had a $14-13-16_{2 C}-6-10$ motif and were therefore classified as ice $A 2 C$ subtypes. Four isolates had the pattern $14-13-16_{2 \mathrm{~B}}-6-13-16_{2 \mathrm{C}^{-}}$ 6-10 and are examples of the ice A2D subtype. These subtypes would be predicted to generate proteins of 94 amino acids but one isolate (Ca102) had an inframe three nucleotide deletion within cassette $16_{2 \mathrm{~B}}$ which resulted in a putative protein truncation of 93 amino acids. Sequence analysis of a fifth isolate (1715) demonstrated a novel variant of the iceA2D subtype that was typified by the sequence 14-13-16 $6_{2 C}-6-13-16_{2 C}-6-10$ (sequence identity $(71 \%)$ and shared residues (80\%) with iceA2D).

Analysis of the protein signal sequence, secondary structures, and topology for the South African ice $A 2$ variants is provided in table 1 . Of note are the observations that these proteins do not have a typical prokaryote signal sequence

Table 1 Protein characteristics of iceA variants

\begin{tabular}{|c|c|c|c|c|}
\hline & FHP1132 & $H P 27$ & Ca1506 & 1715 \\
\hline Variant & $2 \mathrm{~B}$ & $2 \mathrm{C}$ & $2 \mathrm{D}$ & $2 \mathrm{D}^{\prime}$ \\
\hline Amino acids (n) & 59 & 59 & 94 & 95 \\
\hline Cysteine $(\%) t$ & 0 & 0 & 0 & 1 \\
\hline Proline $(\%) t$ & 0 & 0 & 0 & 0 \\
\hline Topology: $\mathrm{N}$ terminus & "in" & "in" & "in" & "in" \\
\hline Topology: $\mathrm{C}$ terminus & ? & $?$ & ? & $?$ \\
\hline \multicolumn{5}{|l|}{ TopPred } \\
\hline $\operatorname{PTM}(\mathrm{n})$ & None & None & One & One \\
\hline Position $\ddagger$ (score) & & & $58-78(0.758)$ & $23-41(0.615)$ \\
\hline \multicolumn{5}{|l|}{ DAS } \\
\hline PTM (n) & None & One & Two & Two \\
\hline Position & & $32-41$ & $37-40,66-75$ & $31-41,70-76$ \\
\hline Helix $(\%) \dagger$ & 11.7 & 0.0 & 0.0 & 0.0 \\
\hline Sheet $(\%) \dagger$ & 41.7 & 62.7 & 72.3 & 54.2 \\
\hline Other $(\%)+$ & 46.7 & 37.3 & 27.7 & 45.8 \\
\hline Classt & Mixed & All-beta & All-beta & All-beta \\
\hline
\end{tabular}

?, not determined; Positionł, amino acid position; †from JPRED.

"in", orientation inside cell membrane; PTM, putative transmembrane segment; DAS, dense alignment sequence. (from the SignalP server), and the $\mathrm{N}$ terminus appears to be embedded within the cell membrane. The TopPred topology program demonstrated that variants $2 \mathrm{~B}$ and $2 \mathrm{C}$ had no predicted transmembrane regions while $2 \mathrm{D}$ and the novel $2 \mathrm{D}$ variant (2D') had a single putative transmembrane region with scores of 0.758 and 0.615 , respectively (fig 4). ${ }^{19}$ The dense alignment surface (DAS) method confirmed that variant $2 \mathrm{~B}$ had no transmembrane region but suggested a possible transmembrane region in $2 \mathrm{C}$ and confirmed two putative transmembrane regions in $2 \mathrm{D}$ and the $2 \mathrm{D}^{\prime}$ variant. ${ }^{20}$ Variant $2 \mathrm{~B}$ can be classified as a mixed class protein but $2 \mathrm{C}, 2 \mathrm{D}$, and $2 \mathrm{D}^{\prime}$ are all all beta proteins.

CLINICAL RELEVANCE OF $i c e A$ GENOTYPES

After validating the PCR protocol and examining the structure of iceA1 and iceA2, PCR was performed in clinical isolates and products were analysed in terms of the distribution of genotypes. Fifty four isolates were positive for either $i c e A 1$ or $i c e A 2$ using previously described primers $I c e A 1 F / 1 R$ and $I c e A 2 F / 2 R,{ }^{5} 54$ isolates were positive for both ice $A 1$ and ice $A 2$, and one isolate did not yield any PCR product for either of the iceA genotypes. Amplicons of the respective sizes were obtained from reference strains 26695 and J99 as expected. Twenty one of the 54 ice $\mathrm{Al}^{+} / \mathrm{ice} A 2^{+}$isolates were re-examined with different ice $A$ primer sets (F5/R4 (iceA1) and F6/R5 $(i c e A 2))^{67}$ to determine whether this "mixed genotype" was a consistent finding. All $21(100 \%)$ were positive for both genotypes, indicating that these primer sets are concordant. These isolates each had a single vacA allele, a single cag $A$ 3' amplicon, which suggested the presence of multiple $i c e A$ genotypes in these strains.

We then examined the presence of these putative "mixed genotypes" further by performing repeat PCR (using two primer sets) and Southern hybridisation in multiply recultured single colonies from eight ice $A 1^{+} / i c e A 2^{+}$ parent strains. Seven of the eight isolates had a single ice $A$ allele by both PCR and Southern analysis (fig 5). No hybridisation was seen in one isolate. A single PCR band using primer sets IceA2F6/IceA2R5 (which identifies iceA2 sizes and subtypes) and a single Southern band was seen in the three clonal strains that were ice $\mathrm{A2}^{+}$. These data signify the presence of multiple $i c e A$ strains rather than mixed genotypes within a single strain in these cultures.

The finding that the $54 i c e A 1^{+} / 2^{+}$strains were mixed cultures suggested identification of both ice $\mathrm{A1}^{+}$and $i c e \mathrm{~A}^{+}$strains from a single gastric biopsy. Fifty four (63\%) of the 86 patients were therefore infected with a single ice $A$ strain. Examination of the distribution of ice $A$ alleles in patients with single strains demonstrated that the distribution differed between the clinical groups $\left(\chi^{2} 7.13, \mathrm{p}<0.03\right)$. Specifically, significantly more patients with GC (10 (67\%) of 15) were infected with iceA1 strains compared with five $(23 \%)$ of 22 with gastritis alone $(p<0.01)$ (fig 6$)$. This was not different to patients with PUD (seven (41\%) of $17 ; \mathrm{p}>0.1$ ). 

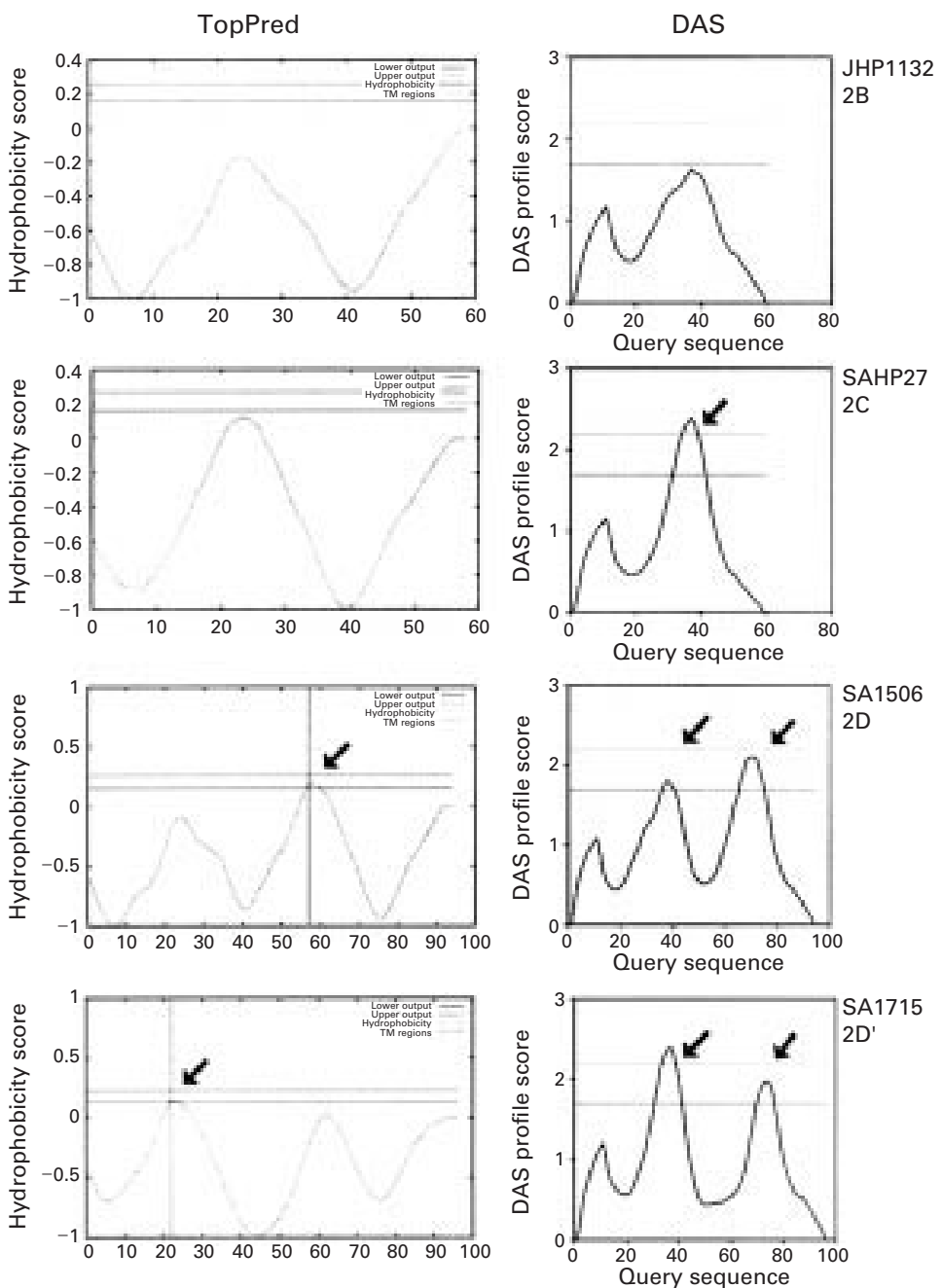

Figure 4 Topology profiles of four iceA2 variants. Left: protein predictions from the TopPred program; right: predictions from the dense alignment sequence (DAS) program. The type strain FHP1132 (iceA2B variant) is indicated in the top panel and South African strains are included with the ice $A 2$ variants signified (2C-2D'). Arrows indicate putative transmembrane regions.

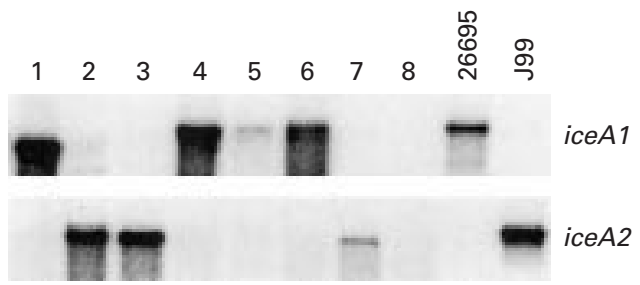

Figure 5 Southern analysis of putatively clonal isolates derived from eight parent strains (lanes 1-8) that were originally ice $\mathrm{Al}^{+} / 2^{+}$by polymerase chain reaction. Four were ice $A 1^{+}$, three ice $A 2^{+}$, and one had no hybridisation. Positive controls (lanes 9 and 10) were 26695 (ice A1 $1^{+}$) and $\mathcal{7 9 9}\left(\right.$ ice $\left.\mathrm{A2}^{+}\right)$.

Examination of the distribution of $i c e A$ genotypes in all 86 patients demonstrated a significant relationship $(p<0.05)$ between infection with ice $A 2$ strains and patients with gastritis alone $(86 \%)$ and PUD (77\%) compared with patients with GC $(47 \%)$.

DISTRIBUTION OF iceA2 SUBTYPES

The size and subtype of the ice $A 2$ alleles were next investigated using primer set IceA2F6/ IceA2R5. As previously reported, ${ }^{67}$ most isolates could be divided into two types according

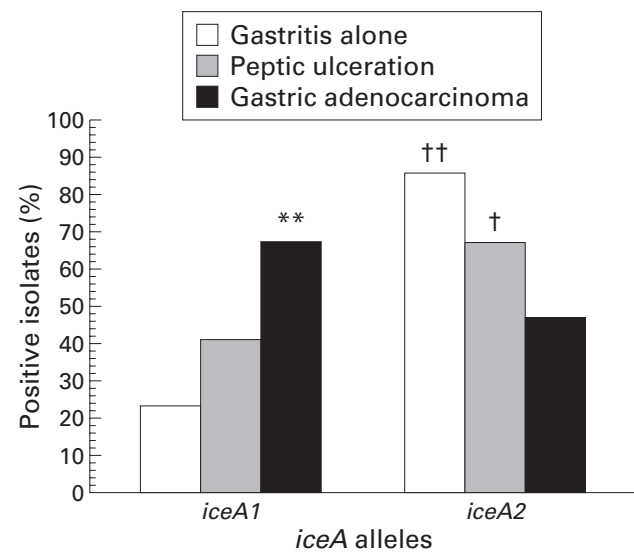

Figure 6 Distribution of iceA genotypes in all isolates. ${ }_{\star \star}^{*} p<0.01$ versus gastritis alone; $t p<0.04,+t p<0.003$ versus gastric cancer.

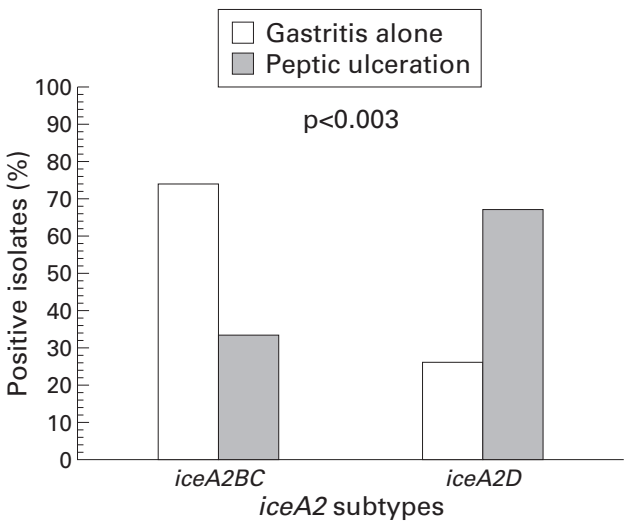

Figure 7 Distribution of iceA2 subtypes in patients with gastritis alone or peptic ulcer disease.

to the presence of 105 nucleotide repeat sequences which resulted in PCR products of either $\sim 229$ bp (iceA2B or iceA2C) or $\sim 334 \mathrm{bp}$ (iceA2D). A number of isolates had either both 229 and $334 \mathrm{bp}$ products or a combination of $229 \mathrm{bp}$ and/or $334 \mathrm{bp}$ and $\sim 49 \mathrm{bp}$ (iceA2E) products. Subsequent analysis of three clonally derived isolates from the latter group demonstrated only single PCR and Southern bands (fig 5), which suggested the existence of multiple ice $A 2$ strains, rather than multiple ice $A 2$ gene copies in these isolates.

Sixty five $(75 \%)$ of 87 patients were infected with a single $i c e A 2$ strain. The distribution of the $i c e A 2$ subtypes was significantly different between the different clinical groups $\left(\chi^{2}\right.$ 9.4, $\mathrm{p}=0.009)$. Specifically, the ice $A 2 B$ or ice $A 2 C$ subtype $(229 \mathrm{bp})$ was predominant in gastritis cases (26 of 35 (67\%); p<0.02 v PUD) (fig 7) while the iceA2D subtype (334 bp) was predominant in PUD cases (14 of $21(67 \%)$ ). No differences in distribution were noted among the 22 patients with multiple $i c e A 2$ subtypes (data not shown).

COMBINATION OF iceA, vacA, AND cagA GENOTYPES All strains were $\operatorname{cag} A^{+}$and gave a single $3^{\prime}$ amplicon. By using the method of van Doorn and colleagues, ${ }^{7}$ eight different combinations based on analysis of the $v a c A$ s region (s1 and $\mathrm{s} 2$ ), and the ice $A$ type (ice $A 1, i c e A 2 B / C$, ice $A 2 D$, and $i c e A 2 E$ ) were examined in all patients (fig 


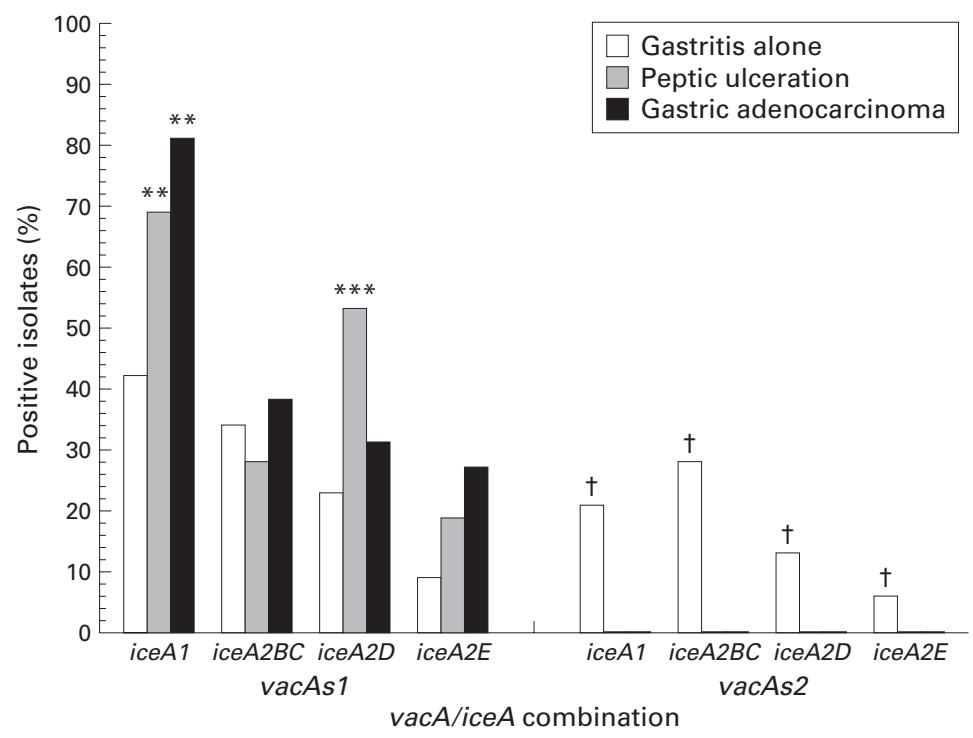

Figure 8 Combination analysis of vacA/iceA alleles and disease outcome in all clinical isolates. ${ }^{\star *} p<0.01,{ }^{\star \star *} p<0.005$ versus gastritis alone; $t p<0.05$ versus peptic ulceration or gastric adenocarcinoma. strains that contained different ice $A$ types (either $i c e A 1 / 2$ or $>1$ ice $A 2$ subtypes). The reason for the high incidence of infection with mixed ice $A$ strains in African patients may simply reflect multiple colonisation but mixed strains do appear to be commonly found and range from $4 \%$ in the $\mathrm{USA}^{9}$ to $15 \%$ in the Netherlands, ${ }^{7} 17 \%$ in Japan, ${ }^{9}$ and $22 \%$ in Colombia. ${ }^{9}$

In the present study, analysis of ice $A$ genotypes in South African patients demonstrated that although ice $A 1$ was more prevalent in PUD patients than in gastritis alone patients, this was not statistically significant. Previous studies in the USA ${ }^{5}$ and the Netherlands ${ }^{7}$ but not in Southeast Asia ${ }^{9}$ have demonstrated a strong association between this allele and PUD. It is possible that the high prevalence $(\sim 50 \%)$ of mixed ice $A$ strains in PUD patients may obscure any potential relationship between the allele and the disease. There was however a strong relationship between the combination of vacA signal sequence subtype and ice $A$ alleles, and PUD. Significantly more PUD isolates were vacA s1/iceA1 compared with $40 \%$ of isolates from patients with gastritis alone.

The function of ice $A 2$ is unknown. In this study, amplicon size of this gene appeared to discriminate between PUD and gastritis alone. iceA2C (229 bp) occurred in $\sim 62 \%$ of all isolates from South African patients with no clinically significant disease while iceA2D (334 $\mathrm{bp)}$ was more prevalent in isolates from patients with PUD. Combination vacA/iceA analysis demonstrated that the vacAs1/ice $A 2 D$ was more prevalent in PUD patients (53\%) than in patients with gastritis alone $(23 \%)$. Consistent with these findings we have recently reported a significant relationship between the cassette structure of $i c e A 2$ and expression in vivo; ice $A 2 A / B / C$ mucosal transcript levels were higher than $i c e A 2 D$ levels. ${ }^{21}$ This suggests that downregulation of ice $A 2$ expression, in addition to induction of ice $A$, may also contribute to disease outcome. It is possible therefore that South African strains from patients with clinically significant disease may not readily express $i c e A 2$ as the majority of these isolates encode the ice $A 2 D$ variant. Alternatively, alterations in the number of repeat cassettes may result in potential changes in protein secondary structure. For example, variants $2 \mathrm{~A}$ and $2 \mathrm{~B}$ may exist as a single globular entity outside the cell while variants $2 \mathrm{C}$ and $2 \mathrm{D}$ are predicted to display one and two surface exposed regions, respectively. These changes may translate into differential binding and/or function of the protein.

There is a paucity of investigations of ice $A$ alleles in isolates from patients with GC. In the one small study, gastric cancer isolates from Japan and Korea were distinguished by the prevalence of $i c e A 1(67 \%)$ while $75 \%$ of cancer isolates from the USA were iceA2. ${ }^{9}$ The numbers in this study were relatively small which limited statistical analysis. In a second Japanese gastric cancer study, ice $A 1$ was associated with enhanced gastric inflammation but not adenocarcinoma per se. ${ }^{8}$ South African $H$ 
pylori cancer isolates, similar to Southeast Asian strains, were distinguished by the presence of $i c e A 1$ and the absence of $i c e A 2$. In contrast however the prevalence of these alleles in cancer patients was significantly different to that found in patients without clinically significant disease in this study. In addition, gastric cancer isolates, unlike those from South African patients with PUD or gastritis, were not distinguished by any specific ice $A 2$ subtype. These data were strengthened when analysed in combination with the vacA status of the strains. Significantly more gastric cancer isolates were distinguished by vacA s1/ice $A 1 \mathrm{com}-$ pared with patients without disease. The putative role of IceA1 in gastric cancer is not defined but experimental studies have demonstrated that long term colonisation in both mouse and monkey models of experimental $H$ pylori infection require a wild-type ice $A 1$ strain. ${ }^{62}$ In the present study, ice $A 1$ does not encode a restriction enzyme and probably, as has been previously suggested, ${ }^{14}$ acts as a transcriptional regulator for downstream genes. It is however possible that the higher percentage of $i c e \mathrm{Al}^{+}$strains in South African gastric cancer patients may simply reflect the observation that these patients have been potentially infected for a longer time period. Alternatively, analysis of differences in iceA1 gene transcription and therefore protein production may reveal a functional role for this protein in this condition. ${ }^{14}$

In summary, the sequences and prevalence of iceA1 and iceA2 in South African $H$ pylori isolates has been investigated, the observation that $\sim 40 \%$ of patients are infected with mixed strains made, and a novel ice $A 2 D$ subtype identified. The potential association between $i c e A 1$ and GC, ice A2D and PUD, and iceA2C and gastritis alone has been demonstrated in our study populations. No isolate from patients with clinically significant disease exhibited either the vac $A$ s $2 / i c e A 1$ or $v a c A$ s $2 / i c e A 2$ allele. Overall, these data support the hypothesis that there is a difference between organisms associated with and without disease. Absolute separation cannot be made which suggests that other factors must play a role in disease pathogenesis. Analysis of $i c e A$ allelic types however is useful in South Africa and certain combinations of virulence factors may provide excellent negative markers for disease.

This study was partially funded by a David and Freda Becker Trust Award (to JAL) and by the National Institutes of Health Grant DK 02381 (to RMP). MK is a recipient of the Claude
Harris Leon Foundation Fellowship. The two reference strains, 26695 and J99, were kind gifts of Dr JC Atherton, Division of Gastroenterology and Institute of Infectious Diseases, University of Nottingham, UK. Ms T Marks performed a number of the PCR reactions.

1 Warren J. Unidentified curved bacilli on gastric epithelium in active chronic gastritis. Lancet 1983;i:1273.

2 Wotherspoon A, Ortiz-Hidalgo $\mathrm{O}$, Falzon $\mathrm{M}$, et al. Helicobacter pylori-associated gastritis and primary B-cell gastric lymphoma. Lancet 1991;338:1175-6.

3 Parsonnet J, Friedman G, Vandersteen D, et al. Helicobacter pylori infection and the risk of gastric carcinoma. $N$ Engl $\mathcal{F}$ Med 1991;325:1127-31.

4 Atherton J. The clinical relevance of strain types of Helicobacter pylori. Gut 1997;40:701-3.

5 Peek R, Thompson S, Donahue J, et al. Adherence to gastric epithelial cells induces expression of a Helicobacter pylori gene, ice $A$, that is associated with clinical outcome. Proc Assoc Am Physicians 1998;110:531-44.

6 Figueiredo C, Quint W, Sanna R, et al. Genetic organization and heterogeneity of the iceA locus of Helicobacter pylori. Gene 2000;246:59-68.

7 van Doorn L, Figueiredo C, Sanna R, et al. Clinical relevance of the cagA, vacA and iceA status of Helicobacter pylori. Gastroenterology 1998;115:58-66.

8 Nishiya D, Shimoyama T, Fukuda S, et al. Evaluation of the clinical relevance of the iceA1 gene in patients with Helicobacter pylori infection in Japan. Scand $\mathcal{f}$ Gastroenterol 2000; 35:36-9.

9 Yamaoka Y, Kodama T, Gutierrez O, et al. Relation between Helicobacter pylori ice $A, \operatorname{cag} A$, and vacA status and clinical outcome: Studies in four different countries. 7 Clin Microoutcome: Studies in
biol 1999;37:2274-9.

10 Kidd M, Lastovica A, Atherton J, et al. Specific genotypes of Helicobacter pylori vacA and cag $A$ but not the presence of $\operatorname{cag} A$, are associated with gastroduodenal disease in South Africa. Gastroenterology 1999;116:G0928. 11 Kidd M, Lastovica A, Atherton J, et al. Heterogeneity in the
Helicobacter pylori genes vacA and cagA: Associated with gastroduodenal disease in South Africa? Gut 1999;45:499503 .

12 Tomb J-F, White O, Kerlavage A, et al. The complete genome sequence of the gastric pathogen Helicobacter pylori. Nature 1997;388:539-47.

13 Alm R, Ling L-S, Moir D, et al. Genomic-sequence comparison of two unrelated isolates of the human gastric pathogen Helicobacter pylori. Nature 1999;397:176-80.

14 Donahue J, Peek R, van Doorn L-J, et al. Analysis of iceA1 transcription in Helicobacter pylori. Helicobacter 2000:5:1 12.

15 Warnich L, Kotze M, Groenewald I, et al. Identification of three mutations and associated haplotypes in the protoporphyrinogen oxidase gene in South African families with variegate porphyria. Hum Mol Genet 1996;5:981-4.

16 Sambrook J, Fritsch E, Maniatis T. Molecular cloning: A laboratory manual, 2 Edn. Cold Spring Harbor, NY: Cold Spring Harbor Laboratories, 1989.

17 Nielsen H, Engelbrecht J, Brunak S, et al. Identification of prokaryotic and eukaryotic signal peptides and prediction prokaryotic and eukaryotic signal peptides and

18 Cuff J, Barton G. Evaluation and improvement of multiple sequence methods for protein secondary structure prediction. Proteins 1999;34:508-19.

19 von Heijne G. Membrane protein structure prediction, hydrophobicity analysis and the positive-inside rule. $\mathcal{F ~} \mathrm{Mol}$ Biol 1992;225:487-94.

20 Cserzo M, Wallin E, Simon I, et al. Prediction of transmembrane alpha-helices in prokaryotic membrane proteins: the dense alignment surface method. Protein Eng 1997;10:6736.

21 Peek R, van Doorn L, Donahue J, et al. Helicobacter pylori $i c e A$ is transcribed in vivo and ice $A 1$ expression is associated with enhanced mucosal inflammation. Gastroenterology 1999;116:A279.

22 Dubois A, Berg D, Incecik E, et al. Transient and persistent experimental infection of nonhuman primates with Helicobacter pylori: implications for human disease. Infect Immun 1996;64:2885-91. 